\title{
Nuevo Algoritmo para la Construcción de la Envolvente Convexa en el Plano
}

\author{
Oscar Y. Buitrago ${ }^{(1)}$, Andrés L. Ramírez ${ }^{(2)}$, Rodrigo A. Britto ${ }^{(3)^{*}}$ \\ (1) Universidad Militar Nueva Granada, Facultad de Ingeniería, Programa de Ingeniería Industrial, Carrera \\ 11 No.101-80 Bogotá - Colombia (e-mail: oscar.buitrago@unimilitar.edu.co) \\ (2) Universidad de la Salle, Carrera 2 No. 10-70 Bogotá - Colombia (e-mail: andres1729@yahoo.com) \\ (3) Universidad de Los Andes, Facultad de Administración, Calle 21 No.1-20 Bogotá-Colombia. (e-mail: ro- \\ britt@uniandes.edu.co)
}

${ }^{*}$ Autor a quien debe ser dirigida la correspondencia

Recibido Nov. 20, 2014; Aceptado Ene. 26, 2015; Versión final Mar. 6, 2015, Publicado Ago. 2015

\section{Resumen}

En el presente trabajo se presenta una nuevo algoritmo para encontrar la envolvente convexa $C(\mathrm{P})$ para un conjuntos $\mathrm{P}$ de $n$ puntos en $\mathrm{R}^{2}$. Este problema ha sido ampliamente estudiado en la geometría computacional ya que tiene importante aplicaciones en la ingeniera y otros campos del conocimiento. El algoritmo propuesto se basada en búsquedas direccionales de hiperplanos soporte y una variante que incorpora hiperplanos separadores que permiten reducir el número de puntos evaluados, descartando los que son interiores. La aplicación del algoritmo propuesto se ilustra mediante un ejemplo. La complejidad del algoritmo obtenido es $\mathrm{O}\left(\operatorname{máx}\left(n v_{0} ; n v^{0}\right)\right), v_{0} \leq v \leq n$ y $v^{0} \leq v \leq n$, donde $v$ es el número de vértices de la envolvente convexa.

Palabras clave: algoritmo, envolvente convexa, hiperplano soporte, búsqueda direccional

\section{New Algorithm to Construct a Planar Convex Hull}

\begin{abstract}
In this paper we present a new algorithm for finding the convex hull $C(P)$ for $P$ sets of $n$ points in $R^{2}$. This problem has been widely studied in computational geometry and has important applications in engineering and other fields of knowledge. The proposed algorithm is based on a directional search of supporting hyperplanes and a variant which includes separating hyperplanes to reduce the number of evaluated points, ignoring the interior ones. The application of the proposed algorithm is illustrated using an example. The complexity of the algorithm obtained is $\mathrm{O}\left(\max \left(n v_{o} ; n v^{0}\right)\right), v_{0} \leq v \leq n$ y $v^{0} \leq v \leq n$, where $v$ is the number of vertices of the convex hull.
\end{abstract}

Keywords: algorithm, convex hull, supporting hyperplane, directional search 


\section{INTRODUCCIÓN}

Para un conjunto $\mathrm{P}$ de $n$ puntos, la envolvente convexa se define como la intersección de todos los conjuntos convexos que contienen a $\mathrm{P}$. El problema de encontrar la envolvente convexa $C(\mathrm{P})$, dado $\mathrm{P}$, consiste en hallar los puntos pertenecientes al mismo que son vértices de $C(\mathrm{P})$ y forma su frontera.

$$
C(\mathbf{P})=\left\{\sum_{i=1}^{n} \alpha_{i} P_{i} \mid P_{i} \in \mathbf{P}, \alpha_{i} \geq 0, \alpha_{i} \in \mathrm{R}, \sum_{i=1}^{n} \alpha_{i}=1\right\}
$$

En particular, en $\mathrm{R}^{2}$ el problema de la envolvente convexa consiste en encontrar el polígono convexo $C(\mathrm{P})$ más pequeño, tal que todos los puntos de $\mathrm{P}$ estén en su frontera o en su interior. Para su solución se desarrollaron algoritmos conocidos como el de Graham (1972), que permite identificar los vértices del polígono, el recorrido de Jarvis (1973), con el que se obtienen los vértices y aristas de la envolvente convexa y puede ser más rápido que el de Graham, el algoritmo de Preparata y Hong (1977) y el algoritmo CONVEX de Eddy (1977). Para dimensiones superiores, Swart (1985) propone encontrar la envolvente convexa, faceta por faceta, y Seidel (1986) desarrolla un método para la construcción de envolventes convexas en varias dimensiones, con un costo logarítmico por cada una de las caras. Posteriormente, Buckley (1998) publica un algoritmo del tipo divide y vencerás, para el problema en el espacio 4dimensional, Chazelle (1993) diseña un algoritmo determinístico para el cálculo de la envolvente convexa de $n$ puntos en $\mathrm{R}^{\mathrm{m}}$ y Sugihara (1994) propone una aproximación topológica a la envolvente convexa en $\mathrm{R}^{3}$.

Bayer (1999) afirma que el problema de encontrar la envolvente convexa ha sido ampliamente estudiado en la geometría computacional y posee muchas aplicaciones en la ingeniería y otros campos del conocimiento entre las que se destacan: análisis de datos de espectrometría, análisis de clusters, detección de colisiones, cristalografía, reconocimiento de patrones, procesamiento de imágenes, integración numérica, estadística, la metalurgia, la cartografía, etc. Para mostrar su vigencia, en este trabajo se describe brevemente aplicaciones de la envolvente que convexa que aparecen en la literatura reciente. Wilderjans et al. (2013) diseñaron un algoritmo que utiliza envolvente convexa para solucionar el problema de selección de modelos y lo aplican con métodos de análisis de componentes principales y de regresión. Por una línea similar, López et al. (2013) entrenan máquinas de soporte vectorial mediante envolventes convexas y cóncavas para solucionar problemas de clasificación. Posteriormente Zeng et al. (2015) proponen un algoritmo también para entrenamiento de máquinas de soporte vectorial en problemas de clasificación pero bajo el concepto de envolvente convexa flexible.

Existen aplicaciones en el procesamiento de imágenes (alternativas a procedimientos diferentes como el de Leal et al., (2014)) y el análisis envolvente de datos. Youssef y Asari (2013) utiliza la envolvente convexa para la identificación de los contornos de la silueta de una persona dentro del marco de algoritmos de reconocimiento de acciones y movimientos. Por su parte, Soltanifar et al. (2013) la emplean para definir de forma más completa las caras sobre las cuales se encuentran las unidades de decisión Pareto eficientes y de esta forma encontrar un mayor número de referentes de comparación para las unidades ineficientes. Los algoritmos para calcular la envolvente convexa en el plano, también tienen aplicaciones en termodinámica, ciencia de materiales y química. Otonello et al. (2013), los utiliza para el estudio de sistemas multicomponentes, en particular el conformado por los óxidos de calcio, silicio y aluminio, mientras que Zhang et al. (2014), lo hacen para la obtención teórica de estructuras cristalinas en el estudio del comportamiento de boruros de metales de transición. Otra interesante aplicación es la de Voskov et al. (2015), que emplean la envolvente convexa para la construcción de diagramas de fases en complejos sistemas multicomponente. En la Tabla 1 se relacionan las aplicaciones más recientes de la envolvente convexa.

Por lo anterior, se puede apreciar que el problema de encontrar la envolvente convexa de un conjunto de puntos en el plano sigue siendo de interés y se continua trabajando en él, tal como lo muestran la propuesta algorítmica de Cinque, L y Di Maggio (2001), que busca mejorar el desempeño computacional de la conocida marcha de Jarvis (Jarvis, 1973) mediante un diseño paralelo y más recientemente los trabajos de Sadhu, Kumar y Kumar (2013) quienes presentan un algoritmo para la generación aleatoria de polígonos, basándose en una versión modificada de la marcha de Jarvis. Zahid y Ashraful (2013), proponen un algoritmo de aproximación para la envolvente convexa, buscando incrementar la velocidad del proceso y mantener un grado aceptable de precisión (esta disminución de la precisión es válida en algunas aplicaciones tales como la animación de gráficos por computador).

En el presente trabajo se presenta un nuevo algoritmo para la solución del problema de la envolvente convexa en el plano. Inicialmente, se explican los fundamentos del algoritmo para el caso bidimensional, se describen los pasos del algoritmo propuesto y se muestra un ejemplo de aplicación. 
Tabla 1: Aplicaciones recientes de la envolvente convexa en diversas áreas del conocimiento

\begin{tabular}{|c|c|c|}
\hline Aplicación & Descripción de la aplicación & Referencia \\
\hline Química & $\begin{array}{l}\text { Se obtienen diagramas de fases en sistemas multicomponentes a } \\
\text { partir de la generación de una malla de puntos, calculando la } \\
\text { energía libre de Gibbs de cada uno y construyendo la envolvente } \\
\text { convexa de aquellos con mínima energía libre de Gibbs en cada } \\
\text { malla. }\end{array}$ & Voskov et al., (2015) \\
\hline $\begin{array}{l}\text { Máquinas de soporte } \\
\text { vectorial (MSV) }\end{array}$ & $\begin{array}{l}\text { Se define la envolvente convexa flexible y se emplea para } \\
\text { solucionar problemas de clasificación en entrenamiento MSV y se } \\
\text { extiende a problemas de clasificación multiclase. }\end{array}$ & Zeng et al., (2015) \\
\hline $\begin{array}{l}\text { Obtención de estructuras } \\
\text { cristalinas teóricas }\end{array}$ & $\begin{array}{l}\text { Se combinan las relaciones entalpia-presión con la envolvente } \\
\text { convexa para identificar las fases más estables a presión cero. }\end{array}$ & Zhang et al., (2014) \\
\hline $\begin{array}{l}\text { Definición de contornos } \\
\text { de siluetas }\end{array}$ & $\begin{array}{l}\text { Reconocimiento de actividades de personas a través de cámaras. } \\
\text { Se utiliza la envolvente convexa para encontrar el polígono más } \\
\text { pequeño que envuelve una silueta humana determinada. }\end{array}$ & $\begin{array}{l}\text { Youssef, M., Asari, V } \\
\text { (2013) }\end{array}$ \\
\hline $\begin{array}{l}\text { Termodinámica de } \\
\text { sistemas líquidos } \\
\text { multicomponentes. }\end{array}$ & $\begin{array}{l}\text { Ajuste matemático del equipotencial loci a diversas condiciones de } \\
\text { presión y temperatura para describir la topología de sistemas } \\
\text { líquidos del sistema formado por óxidos de calcio, silicio y aluminio. }\end{array}$ & $\begin{array}{l}\text { Otonello et al., } \\
(2013)\end{array}$ \\
\hline Aprendizaje supervisado & $\begin{array}{l}\text { Aplicación del método de Jarvis para identificación de envolventes } \\
\text { convexas y cóncavas con el fin de entrenar máquinas de soporte } \\
\text { vectorial en problemas de clasificación. }\end{array}$ & López et al., (2013) \\
\hline Selección de modelos & $\begin{array}{l}\text { Por medio de la envolvente convexa se identifican los modelos } \\
\text { sobre la frontera de la misma que afectan la bondad de ajuste de } \\
\text { forma significativa. Se ejemplifica con métodos de análisis de } \\
\text { componentes principales y de regresión. }\end{array}$ & $\begin{array}{l}\text { Wilderjans et al., } \\
(2013)\end{array}$ \\
\hline $\begin{array}{l}\text { Análisis envolvente de } \\
\text { datos }\end{array}$ & $\begin{array}{l}\text { Se utilizan envolventes convexas para caracterizar completamente } \\
\text { las caras donde se encuentran las unidades eficientes. De esta } \\
\text { forma se identifican pares de referencia que pueden permanecer } \\
\text { ocultos con los métodos de solución tradicionales. }\end{array}$ & Soltanifar et al., 2013 \\
\hline
\end{tabular}

\section{FUNDAMENTOS DEL ALGORITMO PROPUESTO}

Se describe el nuevo algoritmo para encontrar la envolvente convexa en el plano euclidiano. Se parte del hecho de que todo hiperplano divide el correspondiente espacio en dos semiespacios y que por todo vértice de un poliedro pasa por lo menos un hiperplano soporte.

Definición 1. Dado un conjunto $\mathrm{P}$ de $n$ puntos en $\mathrm{R}^{2}, P_{i} \in \mathrm{P}$. Un hiperplano que pasa por $P_{i}$ y que deja los puntos restantes de $\mathrm{P}$ ubicados en un mismo semiespacio cerrado, se denomina hiperplano soporte.

Definición 2. Si el hiperplano que une los vértices $P_{i}$ y $P_{r}$ de $C(\mathrm{P})$ es un hiperplano soporte, entonces el segmento $\overline{P_{i} P_{r}}$ es una arista de $C(\mathrm{P})$.

Definición 3. Dada $C(\mathrm{P})$, si el hiperplano que une los vértices $P_{i}$ y $P_{r}$ no es un hiperplano soporte, es entonces un hiperplano separador (divide el poliedro delimitado por $C(\mathrm{P})$ en dos poliedros).

Teorema 1. Sea $S \subset R^{m}$ un conjunto convexo cerrado. Sea $z^{*}$ un punto de la frontera de $S$. Entonces existe un vector $\mathrm{m}$-dimensional $\mathrm{p} \neq 0$ tal que $z \in S \Rightarrow \mathrm{pz} \leq \mathrm{pz}$.

Corolario 1. Sea conjunto $\mathrm{P}$ de $n$ puntos en $\mathrm{R}^{2}$. Dado que $C(\mathrm{P})$ y su interior forman un conjunto convexo cerrado $\Rightarrow$ si $P_{v} \in \mathrm{P}$ es un vértice de $C(\mathrm{P})$, existe al menos un hiperplano soporte, que de los puntos de $\mathrm{P}$, pasa sólo por $P_{\mathrm{v}}$.

La demostración del Teorema 1 se encuentra detallada en Weitzman (2000).

Considerando nuevamente el conjunto $\mathrm{P}$ de de $n$ puntos para el que se busca $C(\mathrm{P})$, se define la matriz $\mathrm{A}(\mathrm{n} \times 2)$ cuyas columnas contienen respectivamente las abscisas y las ordenadas de los puntos de A. Para el punto $\left(x_{i}, y_{i}\right)$ se define la matriz $c_{i}(n \times 2)$, haciendo que todos los términos de la primera columna sean iguales a $x_{i} y$ los de la segunda a $y_{i}$ : 


$$
\mathbf{A}=\left[\begin{array}{cc}
x_{1} & y_{1} \\
x_{2} & y_{2} \\
x_{3} & y_{3} \\
\vdots \\
x_{n} & y_{n}
\end{array}\right] \quad \mathbf{c}_{\mathbf{i}}=\left[\begin{array}{cc}
x_{i} & y_{i} \\
x_{i} & y_{i} \\
x_{i} & y_{i} \\
\vdots \\
x_{i} & y_{i}
\end{array}\right]
$$

Nótese que si a todos los puntos de $\mathrm{P}$ se les desplazan sus coordenadas, haciendo que el nuevo origen este en el punto $\left(x_{i}, y_{i}\right)$, se obtiene la matriz de coordenadas trasladadas $\left(A-c_{i}\right)$.

Si el punto $\left(x_{i}, y_{i}\right)$ es un vértice de $C(\mathrm{P})$ debe existir un vector $\vec{u}=\left[\begin{array}{c}\cos \theta \\ \operatorname{sen} \theta\end{array}\right]$ normal a un hiperplano soporte (que de los puntos de $\mathrm{P}$ solo incluye a $\left.\left(x_{i}, y_{i}\right)\right)$, tal que $\left(\mathbf{A}-\mathbf{c}_{\mathbf{i}}\right) \vec{u}$ solo tenga entradas no negativas. Esto es cierto porque para todos los puntos de $\mathrm{P}$ (excepto $\left(x_{i}, y_{i}\right)$ ) su producto punto con el vector $\vec{u}$ tendrá el mismo signo, puesto que estarán en un mismo semiespacio abierto definido por el hiperplano soporte que incluye a $\left(x_{i}, y_{i}\right)$.

En particular, la i-ésima componente de $\left(\mathbf{A}-\mathbf{c}_{\mathbf{i}}\right) \vec{u}$ debe cumplir con $\left(\mathbf{A} \vec{u}-\mathbf{c}_{\mathbf{i}} \vec{u}\right)_{i}=0$, luego la i-ésima entrada de $\mathbf{A} \vec{u}$ debe ser la mínima de $\mathbf{A} \vec{u}$. Es decir, si para algún $\theta$ se tiene que $x_{i} \cos \theta+y_{i} \operatorname{sen} \theta$ es la mínima entrada del vector $\mathbf{A} \vec{u}$, entonces $\left(x_{i}, y_{i}\right)$ pertenece a la frontera que conforma la envolvente convexa de los puntos dados.

Es de notar que si $\vec{u}$ es un vector normal a un hiperplano soporte que pasa por $\left(x_{i}, y_{i}\right)$, entonces $-\vec{u}$ también es un vector normal al mismo hiperplano, entonces también es cierto que si para algún $\theta$ se tiene que $x_{i} \cos \theta+y_{i} \operatorname{sen} \theta$ es la máxima entrada del vector $\mathbf{A} \vec{u}$, entonces $\left(x_{i}, y_{i}\right)$ es un vértice de $\mathrm{C}(\mathrm{P})$.

\section{ALGORITMOS PROPUESTOS}

A partir de los fundamentos descritos se desarrollaron dos variantes de algoritmos para construir la envolvente convexa de una nube de puntos en el plano. Uno en el que se hace una búsqueda direccional y otro en el que se recurre al empleo de hiperplanos separadores para descartar puntos interiores.

Algoritmo de búsqueda de vértices

La versión básica denominada algoritmo de búsqueda de vértices, se basa en una búsqueda direccional que, desde el punto con mayor abscisa permite identificar el vértice adyacente en el sentido de las manecillas del reloj, y partiendo de este, encontrar el siguiente y así sucesivamente hasta retornar al punto de partida, identificando totalmente la envolvente. Con base en el anterior análisis, se desarrolló un algoritmo para buscar los vértices de $C(\mathrm{P})$, dado un conjunto $\mathrm{P}$ de $n$ puntos en $\mathrm{R}^{2}$. A continuación se describen los pasos del algoritmo.

Paso 1. Teniendo en cuenta que los puntos con mayores y menores coordenadas en cada uno de los ejes son necesariamente vértices de $C(\mathrm{P})$ :

Determinar el punto $\left(x_{\max } y_{o}\right)$ cuya abscisa es mayor y denominarlo $\mathrm{P}_{\mathrm{o}}$.

Paso 2. Encontrar la intersección de la función $x_{\max } \cos \theta+y_{o} \operatorname{sen} \theta$ con las funciones $x_{i} \cos \theta+y_{i} \operatorname{sen} \theta$ correspondientes a los $(n-1)$ puntos restantes de coordenadas $\left(x_{i}, y_{i}\right)$. La intersección buscada con el punto $\mathrm{P}_{\mathrm{i}}$ esta en $\theta_{i}=\tan ^{-1}\left(\frac{x_{\max }-x_{i}}{y_{i}-y_{0}}\right)$. Dado que $\tan ^{-1}(x)$ es una función continua y creciente, solo es necesario calcular $\left(\frac{x_{\max }-x_{i}}{y_{i}-y_{0}}\right)$. Determinar $\arg _{\operatorname{minimo}}(i)=\operatorname{minímo}_{i}\left(\frac{x_{\max }-x_{i}}{y_{i}-y_{0}}\right)$, Calcular $\theta_{\min }=\tan ^{-1}\left(\arg _{\operatorname{minimo}}(i)\right)$. punto $\mathrm{P}_{\mathrm{i}}$ correspondiente a $\theta_{\min }$ se denomina $\mathrm{P}_{v(1)}\left(x_{v 1} y_{v 1}\right)$ y es el siguiente vértice de $C(\mathrm{P})$. 
Para los restantes puntos (se pueden excluir los vértices encontrados pero se debe mantener $\mathrm{P}_{0}$ ), seleccionar:

$\arg _{\text {minimo }}(i)=\operatorname{minímo}_{i \neq v(j)}\left(\frac{x_{v(j-1)}-x_{i}}{y_{i}-y_{v(j-1)}}\right) . \quad$ Determinar $\theta_{\min v(j)}=\tan ^{-1}\left(\arg _{\operatorname{minimo}}(i)\right) \mid \theta_{i}>\theta_{\min v(j-1)}$

Denominar $\mathrm{P}_{v(j)}$ al punto correspondiente a $\theta_{\min v(j)}$. Este es el siguiente vértice de $C(\mathrm{P})$.

Si $P_{v(j)}=P_{o}$ se han encontrado todos los vértices de $C(P)$ y se finaliza el algoritmo, de lo contrario se busca un nuevo vértice.

FIN

Con la aplicación de este algoritmo se encuentran uno a uno todos los vértices de la envolvente convexa de P. Si $C(P)$ tiene $v$ vértices, la complejidad del algoritmo es $\mathrm{O}(n v)$. También se puede utilizar el algoritmo para identificar un subconjunto de vértices, que permita simplificar la búsqueda construyendo hiperplanos separadores, tal y como se propone en el algoritmo de búsqueda de vértices con hiperplanos separadores.

Algoritmo de búsqueda de vértices incorporando hiperplanos separadores

La variante del algoritmo incorpora la construcción inicial de un hiperplano separador del poliedro delimitado por $C(\mathrm{P})$, se reduce la complejidad del algoritmo en el caso en que no todos los $n$ puntos son vértices, puesto que se descartan en la búsqueda los puntos interiores identificados.

Paso 1.

Determinar el punto $\left(x_{\max } y_{o}\right)$ cuya abscisa es mayor y denominarlo $\mathrm{P}_{\mathrm{o}}$.

Determinar el punto $\left(x_{\min } y_{\min }\right)$ cuya abscisa es menor y denominarlo $\mathrm{P}_{\mathrm{m}}$. En caso de existir más de un punto con abscisa $x_{\min }$ tomar el que tenga menor valor de la ordenada.

Obtener el hiperplano $\mathbf{p x}=k_{o}=\left[\begin{array}{c}y_{\min }-y_{0} \\ x_{\max }-x_{\min }\end{array}\right] \cdot\left[\begin{array}{l}x \\ y\end{array}\right]=\left(y_{\min }-y_{o}\right) x_{\max }-\left(x_{\min }-x_{\max }\right) y_{o}$ que pasa $\mathrm{P}_{\circ}$ y $\mathrm{P}_{\mathrm{m}}$

\section{Paso 2.}

Para $\mathrm{P} \backslash \mathrm{P}_{\mathrm{o}}, \mathrm{P}_{\mathrm{m}}$, calcular los correspondientes $(n-2)$ productos punto y clasificar cada punto en conjunto Q。 (px $\geq k_{\circ}$ ) o en el conjunto $\mathbf{Q}^{\circ}\left(\mathrm{px}<k_{\circ}\right) .\left|\mathbf{Q}_{o}\right|=q_{0},\left|\mathbf{Q}^{\circ}\right|=q^{\circ}, \mathbf{Q}_{o} \cup \mathbf{Q}^{\circ}=\mathbf{P}$.

\section{Paso 3.}

Teniendo en cuenta solamente los puntos contenidos en cada uno de los conjuntos $Q_{0}$ y $Q^{\circ}$ :

Aplicar el algoritmo de búsqueda de vértices iniciando en $\mathrm{P}_{\mathrm{o}}$ hasta culminar en $\mathrm{P}_{\mathrm{m}}$ para encontrar el conjunto de vértices $\mathbf{v}_{o} \subset \mathbf{Q}_{o},\left|\mathbf{v}_{o}\right|=v_{o}$.

Aplicar el algoritmo de búsqueda de vértices iniciando en $\mathrm{P}_{\mathrm{m}}$ hasta culminar en $\mathrm{P}_{\mathrm{o}}$ para encontrar el conjunto de vértices $\mathbf{v}^{\circ} \subset \mathbf{Q}^{\circ},\left|\mathbf{v}^{\circ}\right|=v^{\circ}$.

Es claro que $v_{o} \leq q_{0} \leq n$ y que $v^{o} \leq q^{o} \leq(n-2)$. FIN

En el Paso 3 se puede incorporar (opcionalmente), una etapa adicional consistente en construir hiperplanos separadores $\mathrm{px}=k_{v(i)}$ (que en ocasiones puede ser un hiperplano soporte) que pasen por $\mathrm{P}_{\mathrm{o}} \mathrm{y}$ por cada

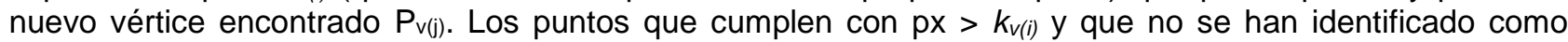
vértices, son interiores y se pueden descartar en las etapas siguientes. La complejidad del algoritmo propuesto es $\mathrm{O}\left(\operatorname{máx}\left(n v_{0} ; n v^{\circ}\right)\right)$ que en el peor de los casos resulta en $\mathrm{O}(n v)$.

Ejemplo de aplicación

Dado el conjunto de puntos $\mathrm{P}_{1}\left(2\right.$ 1), $\mathrm{P}_{2}\left(4\right.$ 0), $\mathrm{P}_{3}\left(-2\right.$ 4), $\mathrm{P}_{4}(1-2), \mathrm{P}_{5}(-4-2), \mathrm{P}_{6}(-2-3), \mathrm{P}_{7}(-3-1), \mathrm{P}_{8}(-4$ 1), $P_{9}\left(-2\right.$ 3), $P_{10}\left(2\right.$ 3), $P_{11}(3-3), P_{12}\left(0\right.$ 4), $P_{13}\left(\begin{array}{ll}-1 & -3\end{array}\right), P_{14}\left(3\right.$ 2), $P_{15}(-1 \quad 1), P_{16}(-1 \quad-5)$, se aplicará el algoritmo para encontrar su envolvente convexa, que como se puede observar en la Fig. 1 tiene como vértices $\mathrm{P}_{2}, \mathrm{P}_{14}$, $P_{10}, P_{12}, P_{3}, P_{8}, P_{5}, P_{16}$ y $P_{11}$. 


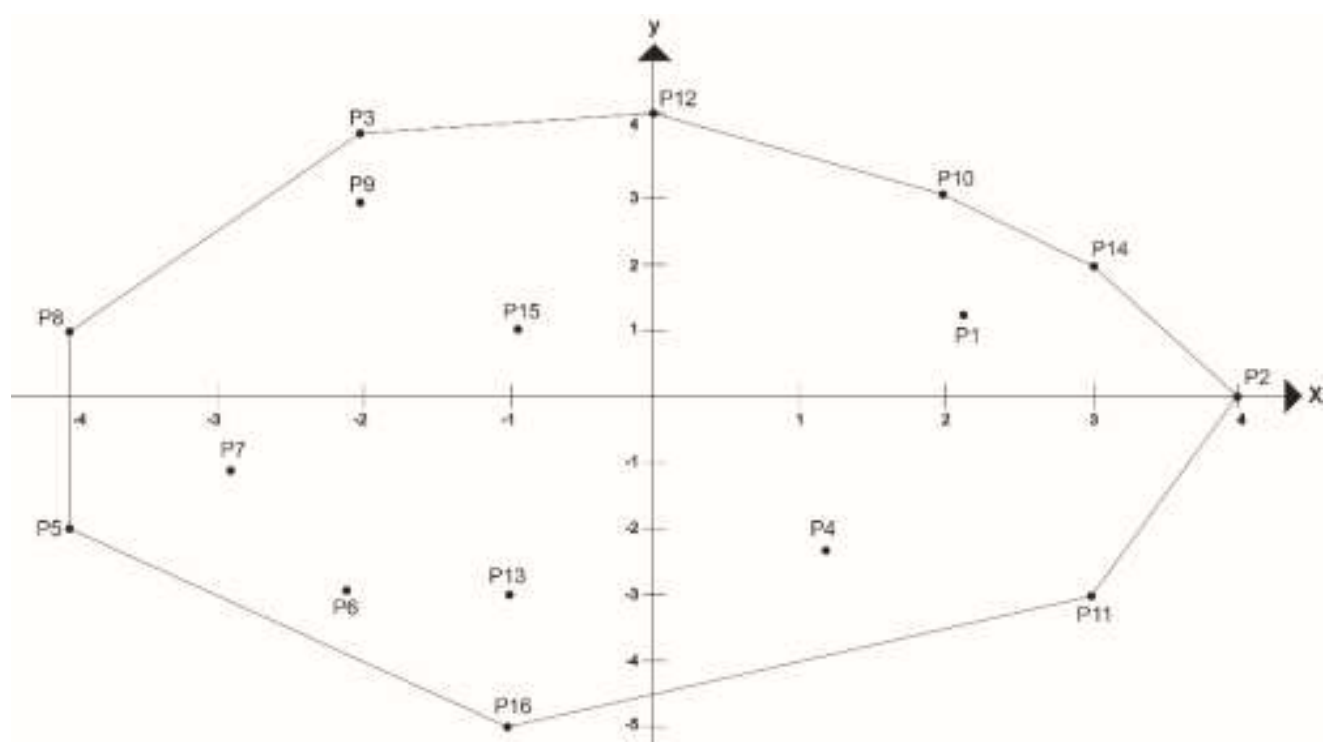

Fig. 1: Envolvente convexa y puntos interiores del ejemplo de aplicación

En la Fig. 2 se grafica la función $x_{i} \cos \theta+y_{i} \operatorname{sen} \theta$ para cada punto $\left(x_{i}, y_{i}\right)$. Se observa que los vértices de la envolvente convexa tienen el valor de su correspondiente función mayor a la de todos los demás en algún intervalo del dominio $0-2 \pi$ radianes. Al seguir la secuencia de los trozos superiores se establece la secuencia en que se conectan los vértices de $C(P) ; P_{2}-P_{14}-P_{10}-P_{12}-P_{3}-P_{8}-P_{5}-P_{16}-P_{11}-P_{2}$.

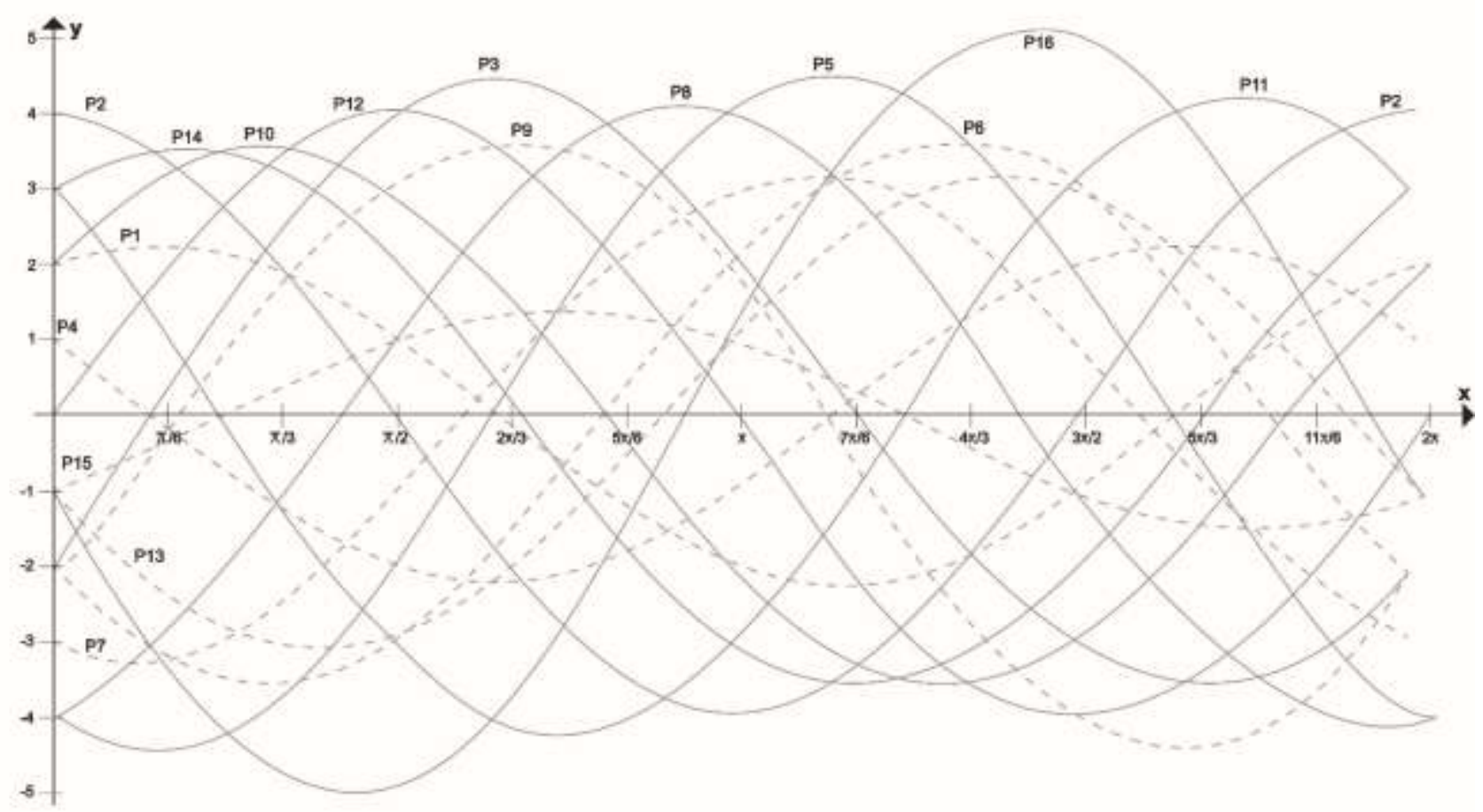

Fig.2: Función $\mathrm{f}(\mathrm{Pi})=x_{i} \cos \theta+y_{i} \operatorname{sen} \theta$ evaluada entre 0 y $2 \pi$ para los puntos del ejemplo.

Para iniciar la aplicación del algoritmo de búsqueda de vértices con hiperplanos separadores, se identifica el punto (4 0), como el de mayor abscisa, por lo tanto $\mathrm{P}_{2}=\mathrm{P}_{\text {o }}$. Por otra parte, existen dos puntos con la menor abscisa $(-4-2)$ y $(-41)$; de estos dos, se elige $P_{5}$ por tener la menor ordenada y se hace $P_{5}=P_{m}$.

El hiperplano que pasa por $\mathrm{P}_{2}$ y $\mathrm{P}_{5}$ es $\mathrm{px}=\mathrm{k}_{0}=\left[\begin{array}{r}-4 \\ 8\end{array}\right]\left[\begin{array}{l}x \\ y\end{array}\right]=-8$. Al hacer los correspondientes productos punto se forma el conjunto $Q_{0}\left(p x \geq k_{0}\right)=\left(P_{1}, P_{2}, P_{3}, P_{5}, P_{7}, P_{8}, P_{9}, P_{10}, P_{12}, P_{14}, P_{15}\right)$ y el conjunto $Q^{\circ}(p x<$ $\left.k_{0}\right)=\left(P_{4}, P_{2}, P_{6}, P_{11}, P_{13}, P_{16}\right)$ 
Se aplica el algoritmo de búsqueda de vértices con los elementos de $Q_{0}$, iniciando en $P_{\circ}\left(\begin{array}{ll}4 & 0\end{array}\right)$. La intersección de $4 \cos \theta+0 \operatorname{sen} \theta$, con las funciones $x_{i} \cos \theta+y_{i} \operatorname{sen} \theta$ de los 10 puntos restantes del conjunto, indican que la primera de ellas está en $\theta_{i}$ es 0,4636 radianes y corresponde a $3 \cos \theta+2 \operatorname{sen} \theta$; es decir, que el siguiente vértice de la envolvente es $\mathrm{P}_{14}\left(\begin{array}{ll}3 & 2\end{array}\right)$. Este punto se excluye para las búsquedas restantes.

A partir de 0,4636 radianes, la siguiente intersección (entre $3 \cos \theta+2 \operatorname{sen} \theta$ y las restantes $x_{i} \cos \theta+y_{i} \operatorname{sen} \theta$ ) está en 0,7854 radianes y corresponde a $\mathrm{P}_{10}(23)$. Ya se tienen tres vértices de la envolvente convexa. Continuando con el procedimiento se identifican como vértices a $P_{12}, P_{3}, P_{8}$ y $P_{m}$. Se descartan los restantes puntos de $Q_{0}$, es decir, que $P_{1}, P_{7}, P_{9}$, y $P_{15}$ no se consideran en adelante.

Ahora, partiendo desde $P_{m}$ se implementa el algoritmo de búsqueda de vértices sobre el conjunto $Q^{\circ}$ y se detecta a $\mathrm{P}_{16}$ como el siguiente elemento de $C(\mathrm{P})$. Nótese que si en esta etapa se construye el hiperplano que pasa por $P_{0}$ y $P_{16}$, se pueden descartar $P_{6}, P_{13}$ y $P_{4}$ como posibles vértices. Continuando la implementación sólo quedan como posibilidades de siguiente vértice $P_{11}$ y $P_{0}$. Se encuentra que $P_{11}$ es un vértice y se culmina la aplicación del algoritmo identificando totalmente la envolvente convexa buscada: $P_{2}$, $\mathrm{P}_{14}, \mathrm{P}_{10}, \mathrm{P}_{12}, \mathrm{P}_{3}, \mathrm{P}_{8}, \mathrm{P}_{5}, \mathrm{P}_{16}$ y $\mathrm{P}_{11}$.

La sola aplicación del algoritmo de búsqueda de vértices también permite obtener la envolvente convexa, pero sin descartar puntos interiores (permite excluir de la búsqueda los vértices que se van encontrando).

\section{CONCLUSIONES}

Se presentó un nuevo algoritmo para la construcción de la envolvente convexa $C(P)$ de un conjunto $\mathrm{P}$ de puntos basándose en búsquedas direccionales de vértices, se dieron los fundamentos teóricos del mismo y se propone una variante mediante la incorporación de hiperplanos separadores, buscando reducir el número de cálculos cuando el conjunto P tiene puntos interiores.

En cuanto a la complejidad, se tiene que para un conjunto de $n$ puntos con $v$ vértices, el algoritmo de búsqueda de vértices (sin incorporar hiperplanos separadores) tiene complejidad $O(n v)$. La variante con hiperplanos separadores tiene complejidad $O$ (máx (nvo; nvo)), con vo $\leq v \leq n$ y $v o \leq v \leq n$.

\section{REFERENCIAS}

Bayer, V, Survey of algorithms for the convex hull problem, Recuperado en 22 de febrero de 2015, de http://citeseerx.ist.psu.edu/viewdoc/download?doi=10.1.1.36.8677\&rep=rep1\&type=pdf (1999)

Buckley, C.E, A divide-and-conquer algorithm for computing 4-dimensional convex hull, International Workshop on Computational Geometry, Wurzburg, March 1988," Lecture Notes in Computer Science, 333, 113-135 (1988)

Cinque, L., Di. Maggio, BSP realisation of Jarvis' algorithm, Pattern Recognition Letters: 22, 147-155 (2001).

Chazelle, B. M, An Optimal Convex Hull Algorithm in Any Fixed Dimension, Discrete and Computational Geometry: 10(1), 377-409 (1993)

Eddy, W. A, new convex hull algorithm for planar sets, ACM Transactions on Mathematical Software (TOMS): 3(4), 398-403 (1977)

Graham, R.L, An efficient algorithm for determining the convex hull of a finite planar set, Information Processing Letter: 1(4), 132-133 (1972)

Jarvis, R. A, On the identification of the convex hull of a finite set of points in the plane, Information Processing Letter: 2 (1), 18-21 (1973)

Leal E., N. Leal., G. Sánchez, Estimación de Normales y Reducción de Datos Atípicos en Nubes de Puntos Tridimensionales, Información Tecnólogica: 25(2), 39-46 (2014)

López, A., X. Li., W. Yu, Convex and concave hulls for classification with support vector machine. Neurocomputing: 122, 198-209 (2013)

Ottonello, G., M. Attene., D. Ameglio., D. Belmonte., D. Vetuschi., M. Natali, Thermodynamic investigation of the $\mathrm{CaO}-\mathrm{Al2O}-\mathrm{SiO} 2$ system at high $P$ and $\mathrm{T}$ through polymer chemistry and convex-hull techniques, Chemical Geology: 346, 81-92 (2013) 
Preparata F. P., S. J. Hong, Convex hulls of finite sets of points in two and three dimensions, Communications of the ACM: 20(2), 87-93 (1977)

Sadhu, S., N. Kumar., B. Kumar, Random Polygon Generation through Convex Layers, Procedia Technology: 10, $356-364$ (2013)

Seidel, R, Constructing higher-dimensional convex hulls at logarithmic cost per face, Proceedings of 18th Annual ACM Symposium on Theory of Computing: 404-413 (1986)

Soltanifar, M., G. R. Jahanshahloo., F. H. Lotfi., S. M. Mansourzadeh, On efficiency in convex hull of DMUs, Applied Mathematical Modelling: 37(4), 2267-2278 (2013)

Sugihara, K, Robust Gift Wrapping for the Three-Dimensional Convex Hull, Journal of Computer and System Sciences: 49(2), 391-407 (1994)

Swart, G, Finding the convex hull facet by facet, Journal of Algorithms: 6(1), 17-48 (1985)

Voskov, A., A. Dzuban., A. Maksimov, TernAPI program for the calculation of ternary phase diagrams withisolated miscibility gaps by the convex hull method, Fluid Phase Equilibria: 388, 50-58 (2015)

Weitzman, M, An 'economics proof' of the supporting hyperplane theorem, Economics Letters: 68(6) 1-6 (2000)

Wilderjans, T.,E. Ceulemans., K. Meers, CHull:A generic convex-hull-based model selection method. Behavior research methods, 45(1), 1-15 (2013)

Youssef, M., Asari, V. Human action recognition using hull convexity defect features with multi-modality setups. Pattern Recognition Letters: 34(15), 1971-1979 (2013)

Zahid, M., M. Ashraful, On Constructing Approximate Convex Hull, International Scholarly and Scientific Research \& Innovation: 7(5), 518-522, (2013)

Zeng, M., Y. Yang., J. Zheng., J. Cheng, Maximum margin classification based on flexible convex hulls, Neurocomputing: 149, 957-965 (2015)

Zhang, X., E. Zhao., Z. Wu., K. Li., Q. Hou, Phase stability and mechanical properties of ruthenium borides from first principles calculations, Computational Materials Science: 95, 377-383 (2014) 